\title{
Passive limb movement intervals results in repeated hyperemic responses in those with paraplegia
}

\author{
Keith J. Burns ${ }^{1} \cdot$ Brandon S. Pollock ${ }^{1} \cdot$ Jon Stavres $\mathbb{1}^{2} \cdot$ Martin Kilbane $^{3} \cdot$ Amber Brochetti $^{3} \cdot$ John $_{\text {McDaniel }}^{3,4}$
}

Received: 10 November 2017 / Revised: 16 February 2018 / Accepted: 18 March 2018 / Published online: 23 April 2018

(c) International Spinal Cord Society 2018

\begin{abstract}
Study design Repeated measures.

Objectives Reports suggest passive limb movement (PLM) could be used as a therapy to increase blood flow and tissue perfusion in the paralyzed lower limbs of those with spinal cord injuries. However, the hyperemic response to PLM appears to be transient, lasting only 30-45 s despite continued limb movement. The purpose of this investigation was to determine whether the hyperemic response is repeatable across multiple short bouts of passive limb movement.

Setting Cleveland Veterans Affairs Medical Center.

Methods Nine individuals with paraplegia $46 \pm 6$ years of age, $17 \pm 12$ years post injury (range: $3-33$ years) with complete T3-T11 injuries were subject to $5 \times 1 \mathrm{~min}$ bouts of passive knee extension/flexion at $1 \mathrm{~Hz}$ with a 1 min recovery period between each bout. Heart rate (HR), mean arterial pressure (MAP), femoral artery blood flow (FABF), skin blood flow (SBF), and tissue perfusion in the lower limb were recorded during baseline and throughout each bout of PLM.

Results Despite no increase in HR $(p \geq 0.8)$ or MAP $(p \geq 0.40)$ across all four bouts of PLM, the average increase in FABF during each bout ranged from $71 \pm 87 \%$ to $88 \pm 93 \%$ greater than baseline $(p \leq 0.043)$. SBF also increased between $465 \pm$ $302 \%$ and $582 \pm 309 \%$ across the five bouts of PLM $(p \leq 0.005)$.

Conclusions Repeated bouts of PLM in those with SCI while in an upright position resulted in a robust and steady increase in FABF and SBF which could have implications for improving vascular health and tissue perfusion in the lower limbs of those with paraplegia.
\end{abstract}

\section{Introduction}

Individuals with spinal cord injury (SCI) are at heightened risk for developing pressure ulcers [1], accelerates the rate of developing cardiovascular disease [2], deep-vein thrombosis, and poor wound healing below the level of the spinal cord lesion. The increased incidence of these conditions is

John McDaniel

JMcDani5@kent.edu

1 Department of Exercise Science, Walsh University, North Canton, $\mathrm{OH}$, USA

2 Heart and Vascular Institute, Penn State College of Medicine, Hershey, PA, USA

3 Advanced Platform Technology Center, Louis Stokes Cleveland Veterans Affairs Medical Center, Cleveland, OH, USA

4 Department of Exercise Physiology, Kent State University, Kent, OH, USA due to decreased physical activity, prolonged periods of immobility, muscle atrophy, changes in muscle fatty composition, and alterations to the structure and function in the peripheral vasculature. For example, previous studies have observed a 30\% reduction in femoral artery diameter [3] and decreases in resting femoral artery blood flow [4] in this population. In addition, changes in vascular and muscle physiology can lead to impairments in blood flow, promote hemorrhage, and increase the rate of intravascular thrombosis and vasospasm [5].

To counteract these detrimental effects, previous investigators have sought to examine the efficacy of utilizing passive leg movement (PLM) to invoke an increase in blood flow to the limb. In an able-bodied population, PLM elicits $\mathrm{a} \approx 45 \mathrm{~s}$ transient hyperemic response in the femoral artery [6]. Both central and peripheral mechanisms are likely responsible for the hyperemia with PLM in able-bodied individuals including stimulation of cardiovascular control center by type III afferent feedback [7], increased stroke volume due to skeletal muscle pump [8], as well as nitric 
oxide [9] and mechanically induced vasodilation [10]. However, due to the nature of a complete SCI afferent feedback to the cardiorespiratory control center is absent, thus the hyperemic response to a bout of PLM would be attributed solely to peripheral factors in this population. Still some investigators [11-13], but not all [14, 15] have reported a significant increase in blood flow to the paralyzed limbs with passive movement. Most recently, Venturelli and colleagues performed continuous blood flow monitoring during a 2 min bout of PLM (knee extension/flexion at 1 $\mathrm{Hz}$ ) and reported a twofold transient increase in femoral artery blood flow despite the absence of a concomitant increase in heart rate.

As the hyperemic response appears to be short lived, lasting $\sim 45 \mathrm{~s}$ despite continued PLM, it likely does not provide a significant shear stress stimulus along the vessel walls to maintain or improve vascular and tissue health. However, Hellsten et al. [16] reported an increases in VEGF protein, endothelial cell proliferation, and eNOS mRNA following a continuous $90 \mathrm{~min}$ bout of PLM potentially due to the repeated mechanical stimulus of lengthening and shortening of the muscle itself. Therefore, if repeated bouts of PLM movement elicit repeated robust hyperemic responses, and subsequently shear stress stimulus, it is likely that the production of pro-angiogenic factors would be augmented and vascular health and tissue perfusion improved. To this end, the primary aim of this study was to evaluate the efficacy of five $1 \mathrm{~min}$ bouts of PLM, with $1 \mathrm{~min}$ recovery periods, to initiate a repeatable and robust hyperemia in the passively moved limb. We hypothesized that each $1 \mathrm{~min}$ bout of PLM would elicit near identical hyperemic responses.

\section{Methods}

Nine individuals with paraplegia participated in the preset study (Table 1). All subjects had clinically confirmed complete lesions (American Spinal Injury Association Impairment Scale level A) between the 3rd and 11th thoracic spinal cord segment (T3-11). All procedures were approved by the Institutional Review Board at the Louis Stokes Cleveland Veterans Affairs Medical Center and written informed consent was obtained from all subjects prior to their participation. Subjects were free from known pulmonary, cardiovascular and metabolic disease, and had a minimal level of spasticity such that it would not interfere with the experimental protocol. Subjects were asked to maintain their current medication regimen but refrain from exercise for $24 \mathrm{~h}$ before reporting to the laboratory and to report in a fasted state $(4 \mathrm{~h})$ with no consumption of caffeine within the previous $8 \mathrm{~h}$.

\section{Experimental protocols}

Subjects were assisted into a comfortable seated-upright chair associated with the Biodex system 4 Pro (Biodex Medical Systems, Shirley, NY, USA) and remained in this position for the duration of the study. The Biodex was calibrated to move the subject's right leg through an $80^{\circ}$ range of motion $\left(10^{\circ}\right.$ to $90^{\circ}$ of knee joint flexion) at an angular velocity of $180 \%$ s ( $\sim 50$ extension/flexions per min). The dynamometer was programmed to cycle at $1 \mathrm{~min}$ intervals ( $1 \mathrm{~min}$ on, $1 \mathrm{~min}$ off) for total of five bouts of PLM. For the duration of the protocol, the subject's contralateral leg remained stationary.

\section{Central hemodynamics}

Heart rate (HR) was determined with the use of a three lead electrocardiogram (ECG) streaming into a data-acquisition box (Lab Chart 8 Pro, AD Instruments, Denver, CO, USA). Mean arterial pressure (MAP) was determined with the use of a Nexfin (Nexfin, Bmeyecorp, Amsterdam, Netherlands). Before the start of data collection, the Nexfin was allowed adequate time for self-calibration (physical) and the finger cuff remained inflated for the entire duration of the protocol. Nexfin's heart reference system was also utilized to correct the hydrostatic pressure differences due to differences in height between the finger and the heart.

\section{Femoral artery blood flow}

Measurement of femoral artery vessel diameter and blood velocity were taken distal to the inguinal ligament and at least $3 \mathrm{~cm}$ proximal to the deep/superficial femoral bifurcation with the use of a Logic 7 ultrasound system (General Electric Medical Systems, Milwaukee, WI, USA). The ultrasound system was equipped with a M12L transducer operating at a frequency of $14 \mathrm{MHz}$ (ultrasound) and $5 \mathrm{MHz}$ (Doppler). Femoral diameter was measured at a $90^{\circ}$ angle along the central axis of the vessel on several images during baseline. These baseline diameter measurements were used to calculate blood flow throughout the entire protocol. Velocity measurements were obtained with the transducer positioned to ensure an insonation angle of $60^{\circ}$ or less, and maintained constant throughout baseline and all five bouts of PLM. Mean blood velocity and arterial diameter were then combined to calculate femoral artery blood flow (FABF) in milliliters per minute with the following equation: mean blood velocity $\times \pi(\text { vessel diameter } / 2)^{2} \times 60$. All scanning and analyses were performed by experienced and skilled sonographers. 
Table 1 Subject characteristics

\begin{tabular}{llllllllll}
\hline Subject & Level & Post injury (years) & Gender & Height $(\mathrm{m})$ & Weight $(\mathrm{kg})$ & BMI & Age (years) & BP $(\mathrm{mmHg})$ & Medication \\
\hline 1 & T7 & 25 & F & 1.60 & 54.1 & 21 & 55 & $116 / 64$ & $10 \mathrm{mg}$ baclofen \\
2 & T9 & 4 & M & 1.78 & 104.5 & 33 & 44 & $126 / 80$ & $5 \mathrm{mg}$ diazepam \\
3 & T7 & 32 & M & 1.75 & 64.1 & 21 & 53 & $118 / 64$ & $5 \mathrm{mg}$ diazepam \\
4 & T1 & 33 & M & 1.80 & 73.6 & 23 & 53 & $132 / 76$ & -- \\
5 & T7 & 16 & M & 1.78 & 88.2 & 28 & 44 & $114 / 70$ & -- \\
6 & T8 & 28 & M & 1.75 & 80.5 & 26 & 53 & $130 / 80$ & $5 \mathrm{mg}$ baclofen \\
7 & T1 & 14 & M & 1.75 & 105.5 & 34 & 40 & $132 / 76$ & $10 \mathrm{mg}$ baclofen \\
8 & T9 & 3 & M & 1.75 & 89.5 & 29 & 49 & $112 / 76$ & -- \\
9 & T3 & 3 & F & 1.72 & 79.1 & 27 & 40 & $106 / 68$ & -- \\
Average & - & $17 \pm 12$ & - & $1.74 \pm 0.1$ & $82.1 \pm 17.1$ & $27 \pm 5$ & $48 \pm 6$ & $121 / 73$ & -- \\
\hline
\end{tabular}

\section{Tissue oxygenation}

Changes in tissue oxy-hemoglobin (Oxy), total hemoglobin $(\mathrm{tHb})$, and normalized tissue hemoglobin index (nTHI) [17], the percent change from initial hemoglobin concentration, were assessed with the use of a NIRS (near infrared spectroscopy) system (NIRO-200, Hamamatsu Phototonics, Hamamatsu, Japan) by placing electrodes on the anterior aspect of the rectus femoris muscle. The laser on the NIRS system penetrates $\sim 2 \mathrm{~cm}$ into the muscle tissue. Total hemoglobin was calculated from alterations in light absorption at each of the transmitter laser frequencies according to the Beer-Lambert law. Rather than utilizing an estimated optical pathlength, the NIRO-200 provided delta values relative to pathlength $(\Delta \mu \mathrm{M} / \mathrm{cm})$. Prior to the start of baseline recordings the NIRs unit was 'zeroset' allowing us to simply report changes in $\mathrm{Oxy}, \mathrm{tHb}$, and nTHI from the initial baseline to the end of each bout of PLM.

\section{Skin blood flow}

Skin blood flow (SBF) was determined via a Laser Doppler and Perfusion Monitor (moorVMS-LDF2, Moor Instruments, Axminster, UK) with the VP1/7 probe placed on the anterior aspect of rectus femoris muscle over the middle of the muscle belly. Laser Doppler flowmetry is based on red blood cell flux and calculated as product of red blood cell concentration and velocity. This variable is expressed in arbitrary units (PU) and is indicative of superficial skin blood flow [18].

\section{Data analysis}

The dependent variables assessed were FABF, SBF, HR, MAP, Oxy, tHb, and nTHI. Throughout the protocol all variables went through analog-to-digital conversion and were simultaneously acquired $(20 \mathrm{~Hz})$ by commercially available data-acquisition system and supporting software (powerlab 8/36 and Lab Chart Pro 8, AD Instruments, Denver, CO, USA). Owing to the different kinetics of each variable, the approach to the analysis was not the same for all variables. With regards to femoral blood flow and skin blood flow the data were averaged into a $1 \mathrm{~min}$ baseline value, $1 \mathrm{~min}$ averages for each of the $1 \mathrm{~min}$ bouts of PLM, and then 1 min recovery following the last bout of PLM. In addition, the peak FABF during each bout of PLM was determined. One-way repeated measures ANOVA was used to determine whether the main effect of PLM bout was significant. If a main effect of bout was present, paired samples $t$-tests were used to simply compare the baseline value to the individual values for each bout of PLM. To determine whether the increase in femoral blood flow was constant throughout each bout of PLM $t$-tests were utilized to compare blood flow during seconds 12-24 (after initial rise) and 48-60. With regards to Oxy, tHb, and nTHI, baseline and recovery values were compared to the values during the last $12 \mathrm{~s}$ of each bout of PLM. Post-hoc comparisons were adjusted using the Benjamini-Hochberg false discovery rate correction equation. An a priori power analysis indicated a sample size of eight subjects would be needed to obtain a statistical significance of $p<0.05$ and power of $\beta>0.80$. All data are presented as mean \pm SD.

\section{Results}

\section{Heart rate and mean arterial pressure}

There were no statistical differences in HR or MAP from baseline to each bout of PLM. At baseline, HR was $82 \pm 13$ bpm while during the five bouts of PLM, the average heart rates were $81 \pm 10,81 \pm 10,82 \pm 11,80 \pm 11$, and $83 \pm 7$ bpm $(p \geq 0.8)$. At baseline MAP was $99.6 \pm 19.2 \mathrm{mmHg}$, while during the bouts of PLM MAP averaged 101.0 \pm 17.1 , $104.1 \pm 17.7,103.4 \pm 16.4,101.8 \pm 18.6$, and $106.7 \pm 16.8$ $\mathrm{mmHg}(p \geq 0.4)$. 

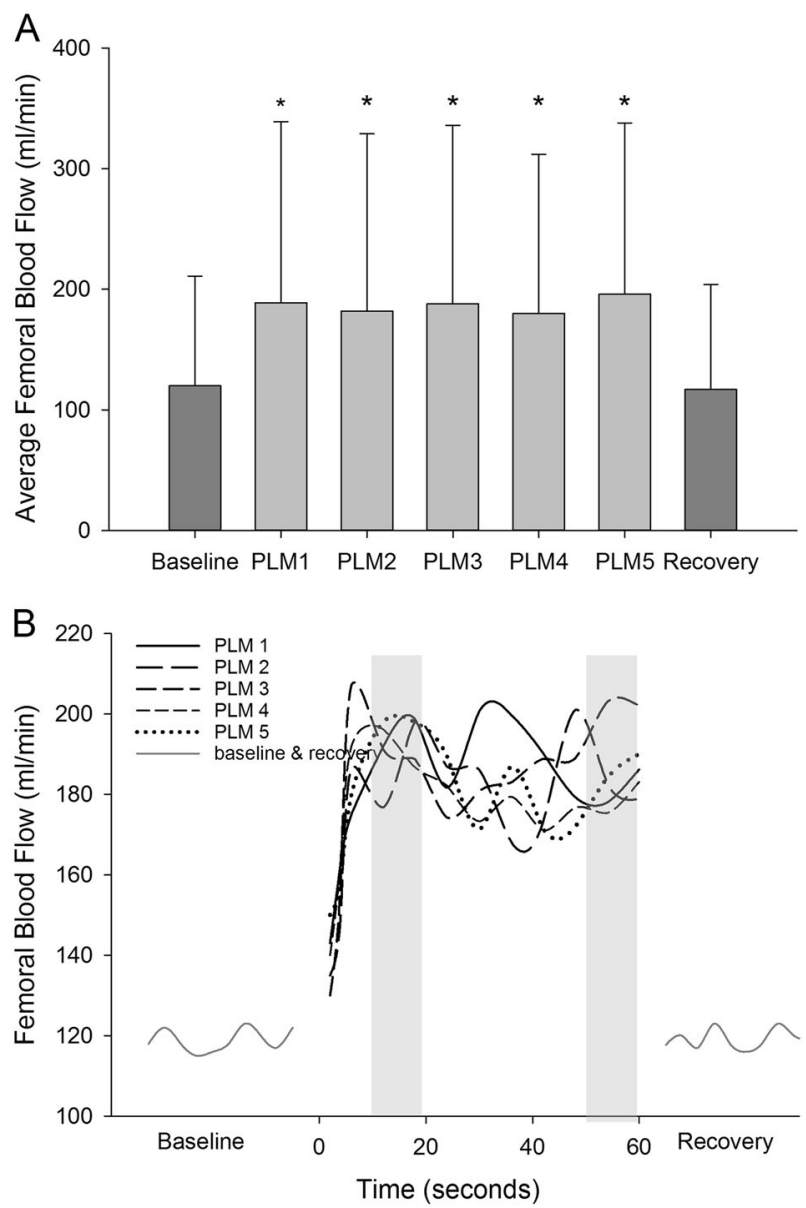

Fig. 1 Average femoral blood flow values across the five bouts of PLM. *Denotes a significant increase $(p<0.05)$ from baseline and recovery (a). There was no difference in average blood flow between the five bouts of PLM. Data presented as mean \pm SD. Line graphs illustrating the kinetics of femoral blood flow for each bout of PLM (b). $T$-tests revealed no significant differences in average blood flow between $12-24$ and $48-60 \mathrm{~s}$ (represented by gray column) within each bout of PLM

\section{Femoral artery blood flow}

Repeated measures ANOVA revealed a main effect of bout $(p=0.03)$ for the average femoral blood flow. At baseline, FABF was $120 \pm 91 \mathrm{ml} / \mathrm{min}$ and the average during each subsequent $1 \mathrm{~min}$ bout of PLM was $189 \pm 150 \mathrm{ml} / \mathrm{min}(85 \pm$ $103 \%), \quad 182 \pm 147 \mathrm{ml} / \mathrm{min}(71 \pm 87 \%), \quad 188 \pm 148 \mathrm{ml} / \mathrm{min}$ $(79 \pm 90 \%), 180 \pm 132 \mathrm{ml} / \mathrm{min}(76 \pm 87 \%)$, and $196 \pm 142$ $\mathrm{ml} / \mathrm{min}(88 \pm 93 \%)$, respectively (Fig. 1a). These values were all significantly greater than the baseline $(p \leq 0.04)$ and recovery $(123 \pm 90 \mathrm{ml} / \mathrm{min} ; p \leq 0.031)$ but were not significantly different from each other $(p=0.81)$. The effect size ranged from 0.25 to 0.30 . $T$-tests revealed that the average blood flow during seconds 12-24 and 48-60 within each bout of PLM were not significantly different from each other ( $p \geq 0.32$ for all comparisons; Fig. 1b).
Repeated measures ANOVA also indicated a significant main effect of bout for peak FABF $(p<0.001)$. FABF values for each bout of PLM was $253 \pm 196 \mathrm{ml} / \mathrm{min}(174 \pm$ $224 \%), 248 \pm 204 \mathrm{ml} / \mathrm{min}(148 \pm 175 \%), 257 \pm 206 \mathrm{ml} / \mathrm{min}$ $(172 \pm 208 \%), 245 \pm 178 \mathrm{ml} / \mathrm{min}(163 \pm 190 \%)$, and $227 \pm$ $168 \mathrm{ml} / \mathrm{min}(153 \pm 207 \%)$, respectively, which were all significantly different from baseline $(p \leq 0.021)$ but not different from each other $(p=0.405)$. The effect size ranged from 0.37 to 0.40 .

\section{Oxy-hemoglobin, total hemoglobin, and normalized tissue hemoglobin index}

At baseline Oxy was $-0.57 \pm 3.49 \Delta \mu \mathrm{M} / \mathrm{cm}$ and dropped to $-2.79 \pm 2.51(p=0.088),-3.76 \pm 3.01(p=0.042),-3.51$ $\pm 3.30(p=0.068)-3.15 \pm 4.07(p=0.106)$, and $-3.65 \pm$ 4.37 ( $p=0.080)$ during the last $12 \mathrm{~s}$ of each bout of PLM respectively (Fig. 2a). Thus, significant decreases in Oxy were observed only in one bout but strong trends were observed in the rest. The average Oxy over the last $12 \mathrm{~s}$ of each bout of PLM was significantly lower than the Oxy during the recovery period $(-0.10 \Delta \mu \mathrm{M} / \mathrm{cm})$ for all bouts of PLM except the 4th $(p=0.054)$. Baseline tHb was $0.40 \pm$ $4.46 \Delta \mu \mathrm{M} / \mathrm{cm}$ and dropped to $-2.70 \pm 3.78(p=0.113)$, $-4.45 \pm 5.47(p=0.04),-4.00 \pm 5.62(p=0.07),-3.57 \pm$ 6.63 (0.108), and $-4.08 \pm 7.13(0.086)$ at the end of each of the five bouts of PLM, respectively (Fig. 2b). During recovery period $\mathrm{tHb}$ was $1.24 \pm 2.09$ that was significantly greater than $\mathrm{tHb}$ at the end of bout 2 and $3(p \leq 0.38)$ and had strong trends for bouts $1(p=0.053), 4(p=0.078)$, and $5(p=0.063)$. Finally, nTHI at baseline was $0.996 \pm 0.046$ a.u. and fell to $0.917 \pm 0.069,0.905 \pm 0.098,0.899 \pm 0.095$, $0.896 \pm 0.100$, and $0.897 \pm 0.106$ during the last $12 \mathrm{~s}$ during the five bouts of PLM (Fig. 2c). The change from baseline to the end of PLM was significant for all five bouts ( $p \leq$ 0.022). Furthermore, nTHI at the end of each bout of PLM was significantly reduced compared to the recovery (1.018 $\pm 0.036 ; p \leq 0.011$ for all comparisons).

\section{Skin blood flow}

Similar to femoral blood flow, repeated measures ANOVA indicated a significant effect of bout for average skin blood flow ( $p<0.001)$. At baseline SBF was $18.3 \pm 10.0$ PU and the average during each subsequent 1 min bout of PLM was $83.4 \pm 26.0,85.9 \pm 35.7,94.9 \pm 52.2,101.5 \pm 70.8$, and 117 $\pm 87.4 \mathrm{PU}$, which were all significantly greater than baseline $(p \leq 0.005)$ but were not different from each other $(p=$ 0.216 ) (Fig. 3a). The effect size ranged from 0.62 to 0.85 . Skin blood flow during the recovery period was $23.5 \pm 30.1$ PU, which was significantly less than average skin blood flow during each bout of PLM $(p \leq 0.015)$. 

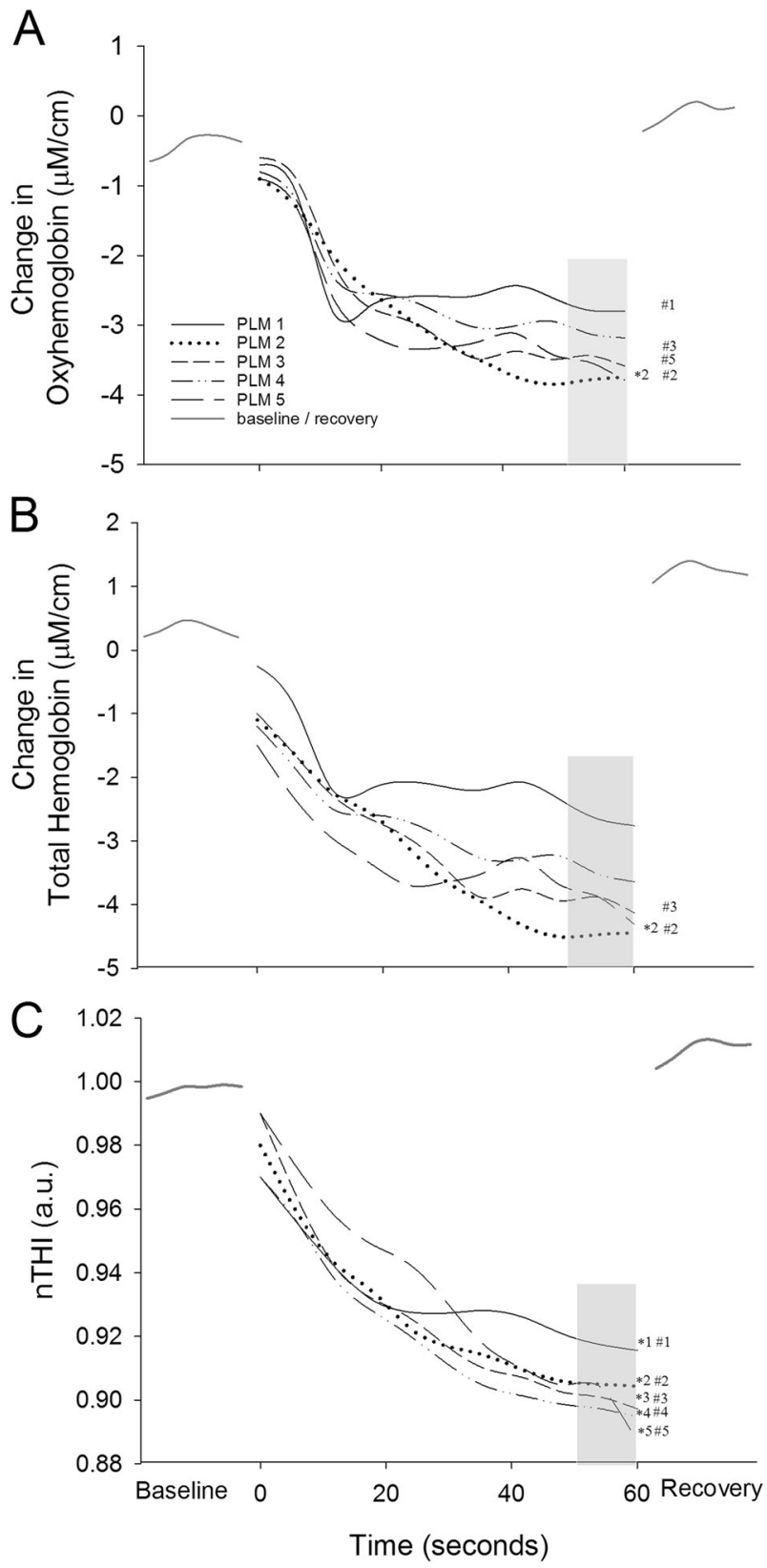

Fig. 2 Changes in oxygenated hemoglobin (a), total hemoglobin (b), and nTHI (c) values at baseline, across the five bouts of PLM and during recovery. *Denotes a significant decrease from the baseline value while "denotes a significant decrease from the recovery period. The numbers next to the symbols indicate which bout of PLM resulted in significant differences $(p<0.05)$. Note, see results as there were strong trends for many of the trials that did not achieve significance ( $p$ $<0.08)$

\section{Discussion}

Previous investigators have reported a transient hyperemic response to PLM. However, the present study is the first to determine whether the hyperemic response can be repeated in those with complete spinal cord injuries when the bouts of PLM are interspaced with short recovery periods.
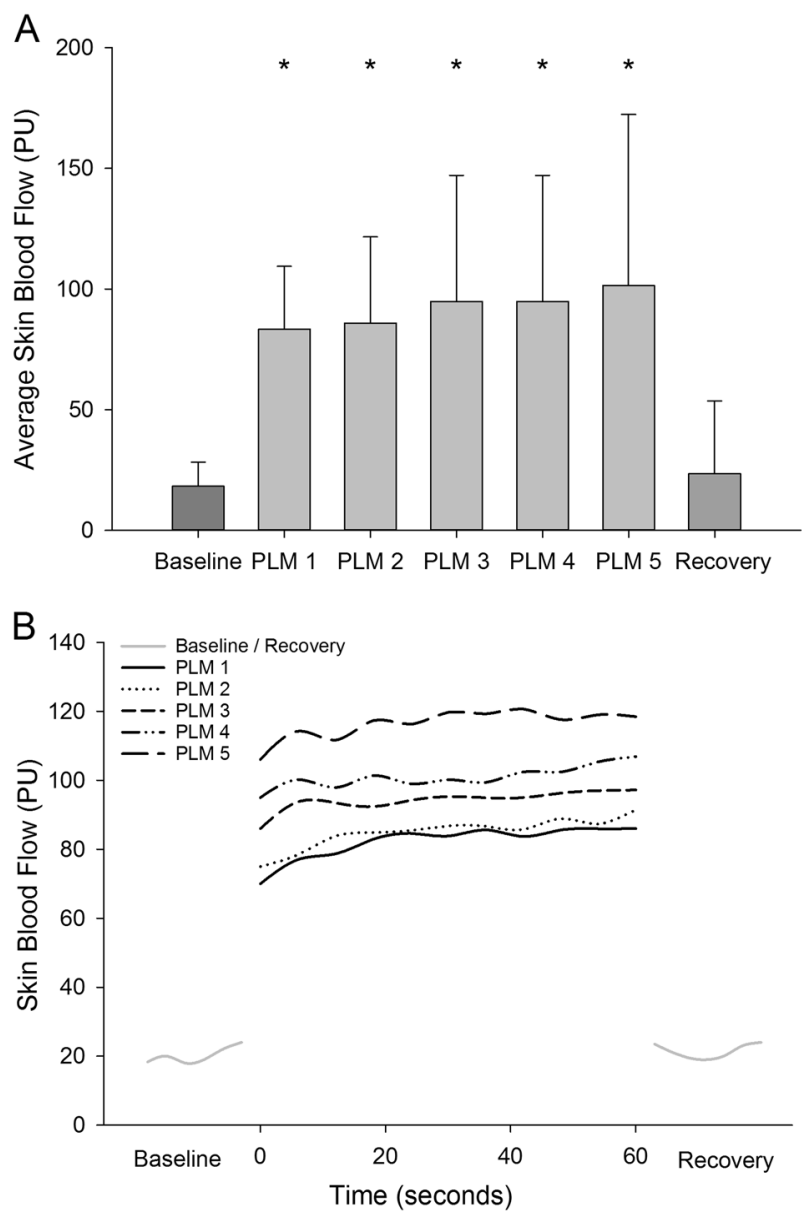

Fig. 3 Average skin blood flow across the five bouts of PLM (a) and line graphs illustrating the kinetics of skin blood flow within those bouts (b). *Denotes a significant increase $(p<0.05)$ from baseline and recovery. Data presented as mean $\pm \mathrm{SD}$. Line graphs illustrating the kinetics of skin blood flow for each bout of PLM

Specifically, we examined whether repeated bouts of PLM could result in consistent changes in FABF, SBF, and tissue perfusion of the passively moved limb. The present study not only indicates that despite no increase in HR or MAP, repeated $1 \mathrm{~min}$ bouts of PLM results in repeated robust increases in FABF, but also that when those with SCI are placed in an upright position the hyperemic response is constant rather than transient. This suggests that utilizing PLM could be used to stimulate greater hyperemic responses and ultimately vascular shear stress in the SCI population. This has implications for the use of passive movement as a rehabilitation modality to promote vascular health and angiogenesis.

\section{Hyperemic responses}

Several investigators have reported there is no increase in FABF during passive movements in the SCI population $[14,15]$ while others have reported passive movements do 
increase blood flow or blood velocity [11-13]. Specifically, this hyperemic response was not observed by Svensson et al. [15] and Ter Woerds [14] as they measured blood flow upon completion of the passive movements rather than during the movements and likely missed the hyperemic response. The results of the current study agree with the studies performed by Ballaz and Venturelli. Specifically, Venturelli et al. [12,13] reported peak blood flow responses to be nearly double baseline values during a sole bout of PLM. The results of the current study are similar with an average peak blood flow of $162 \%$ above baseline values observed across all five bouts of PLM. Interestingly, the hyperemic response between the able-bodied and SCI population are nearly identical after controlling for thigh volume and resting FABF, therefore it was postulated that those with SCI have heightened vascular sensitivity below the level of the lesion [12]. As this hypersensitivity is likely in response to the non-active paralyzed limb being subjected to unaccustomed movements [19], it was unknown whether or not employing repeated bouts of PLM would diminish this sensitivity resulting in a decreased hyperemic response with each subsequent bout of PLM. The results of the present study are promising as the five bouts of PLM resulted in nearly identical hyperemic responses. A followup study is required to determine whether this response remains after chronic exposure to repeated bouts of PLM.

Surprisingly this investigation the hyperemic responses to all five bouts of PLM were not transient, rather blood flow remained elevated throughout the entire bout of PLM (Fig. 1). This is in stark contrast to previous reports on the able-bodied population $[6,20,21]$ and those with spinal cord injuries $[12,13]$. This discrepancy is likely due to the lack of sympathetic stimulation in the lower legs of our subjects and the upright body position utilized in this investigation. Specifically, Trinity et al. [21] reported a prolonged hyperemic response to PLM when subjects were in an upright position rather than a supine position due to the additional influence of gravity on lower limb blood flow. In addition, the lack of sympathetic control of the vessels in the lower limbs results in excessive blood pooling in the lower extremities especially while in the upright position. The onset of PLM likely results in mechanically induced [10] and nitric oxide stimulated [22, 23] vasodilation and also engages the skeletal muscle pump, albeit to a lower extent than active exercise facilitating venous return and ultimately blood throughput in the lower limb. Thus, with regards to utilizing PLM as a therapy modality to improve blood flow and tissue perfusion in the lower limbs of those with SCI, it appears that circumventing the transient hyperemic response is possible with a more aggressive upright posture rather than a supine position.

Previous reports indicate that at least in the able-bodied population $35 \%$ of the hyperemic response is from central factors and $65 \%$ is the result of peripheral mechanisms [23]. The robust hyperemic responses observed in this investigation are not facilitated by central factors as heart rate and mean arterial pressure did not to change with PLM. This is in agreement with previous studies that looked at heart rates responses during either passive cycling $[11,24]$ or PLM $[12,13]$ in those with spinal cord injuries and also reported no changes in HR. This absence of central responses in the SCI population, particularly in the AIS A subgroup, is likely due to the lack of afferent feedback from the limbs to the cardiovascular control center. This is supported by Trinity et al. [25] that reported significant decrease in HR response to PLM in able-bodied individuals following an intrathecal fentanyl injection that blocks the afferent feedback from the muscles of the lower limb.

The repeatable and prolonged hyperemic response could augment the promotion of a pro-angiogenic environment in the skeletal muscle by increasing the physiologic stimulators of angiogenesis leading to increased vessel growth and capillary density [26]. A recent investigation by Liu et al. [27] reported an engineered transcription factor that activates VEGF leads to an increased capillary density in spinal cord-injured rodent model. This supports that upregulation of the VEGF in human spinal-injured persons could illicit a similar response. An investigation by Hellsten and colleagues reported increases in VEGF protein at 30,60, and 90 min after the start of a 90 min bout of continuous PLM. This increase was attributed to shear stress due to increased blood flow and mechanical stress due to the passive shortening and lengthening of the muscle [16]. Therefore, it is likely that the hyperemic response invoked by the PLM, similar to those performed in the current study, could promote a greater increase in VEGF leading to a more favorable pro-angiogenic environment in the passively moved limb.

\section{Muscle tissue perfusion and skin blood flow}

Unlike FABF, Oxy, tHb, and nTHI did not appear to reach a steady state within the $1 \mathrm{~min}$ bouts of PLM, rather they tended to decrease from baseline over time (Fig. 2). Although the decrease in hemoglobin may seem to contradict the FABF response, this could again be explained by the lack of vascular control in the lower limbs in our subjects. Specifically, the inability to constrict the vessels in the lower limb through sympathetic activation [28] can result in significant pooling of venous blood in the lower limbs when in a seated or standing position in this population [28, 29]. The rhythmic lengthening and shortening of the hamstrings and quadriceps likely promoted venous return from the lower limbs via the skeletal muscle pump resulting in the tendency for $\mathrm{Oxy}, \mathrm{tHb}$, and ultimately nTHI to decrease within each bout of PLM. Ultimately venous return was 
augmented to a greater extent than femoral blood flow reducing hemoglobin concentration but increasing hemoglobin throughput in the muscle. It is likely that if these bouts of PLM continued for longer than 1 min tissue perfusion would have also reached a steady state. Similar to femoral blood flow, skin blood flow was elevated during each bout of PLM and returned to baseline values during the recovery period. Unlike femoral blood flow and tissue perfusion, skin blood flow did not appear to remain steady across each bout and was absent in the onset kinetics. This is likely due to the fact that the skin is not influenced by the skeletal muscle pump.

\section{Implications}

In addition to the benefits to vascular health previously mentioned, the increase in skin blood flow and tissue perfusion could provide other health benefits. Specifically, deep-vein thrombosis (DVT) which is three times higher in the SCI population compared to the able-bodied population [30] is partially due to stasis of blood flow and endothelial injury [31]. Periodic PLM could reduce blood stasis and improve endothelial function. In addition, PLM could prove beneficial at reducing pressure ulcers and improving skin wound healing in the SCI population. Specifically, an investigation by Sonenblum et al. [32] looked at the effectiveness of pressure relief maneuvers to increase skin blood flow of the buttocks reported comparable increases in SBF to the current investigation. Coggrave and Rose [33] reported the mean duration of a pressure lift needed to achieve pressure relief to allow for adequate SBF and tissue oxygenation above the ischial tuberosity was $1 \mathrm{~min}$ and 51

s. Holding pressure lifts for this long of duration could be extremely difficult for persons with paraplegia as they may not possess the upper body strength or motivation required. The prolonged increase in skin and muscle perfusion observed with PLM may help reduce the risk of pressure ulcers. However, the present study measured SBF and tissue oxygenation on the thigh while pressure ulcers most commonly form in areas that are exposed to constant pressure and bony prominences such as the ischial and sacral regions. Thus, although these results may not directly translate to pressure ulcer reduction around the ischial tuberosity, this study does provide motivation for future investigations to evaluate changes in SBF around the ischial tuberosities. Such studies should also include movement across the hip joint.

The decreased physical activity and absence of autonomic regulation following a SCI is a major contributor to cardiovascular disease and subsequent death [34, 35]. Although it is well established that increased physical activity improves central cardiovascular health, it is less clear which exercise modalities improve peripheral vascular function in the paralyzed limbs in this population. Previous report indicated that PLM results in a transient hyperemic response. However, the data gathered in this investigation indicate that the hyperemic response is constant in the SCI population when performed in a more upright posture and support the use of PLM to initiate hyperemic responses in the peripheral vasculature which can facilitate improvement [16] or at least maintenance of peripheral vascular function. Owing to the passive nature of this modality, benefits typically associated with increased muscle metabolic rate of voluntary exercise, such as improved lipid and glucose metabolism, will likely be absent. Other proposed therapeutic modalities for this population include FES stimulation and upper body exercise. Although FES stimulation might be the optimal strategy as it is associated with increased metabolic rate of the paralyzed muscle, not all individuals respond to FES and it is often cost prohibitive or unavailable to the majority of individuals with SCI. Upper body exercise can also lead to improvements in central cardiovascular health. However, reports on the influence of upper body exercise on lower limb blood flow are mixed [4, 29, 36] likely due to the range of autonomic dysregulation in this population. In addition to the mixed results from FES and upper body exercise investigations, the use of both of these modalities are limited to the availability of equipment, which can be costly and difficult to use. A potential benefit to PLM is that no equipment is needed and could be performed on an hourly or daily basis by a care-giver or a therapist and passive nature of the modality may be safer as it has limited chances of evoking autonomic dysreflexia. As previously stated, future studies need to focus on tissue changes in tissue perfusion where PU often occur, and include PLM across the hip joint to include those hip extension muscles that are often impacted by pressure ulcers. Furthermore, this study is only individuals with SCI between T3 and T12. The inclusion of higher level injuries that result in flaccid paralysis also needs to be investigated. Currently we assume that they would have similar hyperemic responses but to a reduced magnitude due to a reduced muscle mass [12].

\section{Conclusion}

Previous studies have observed that the hyperemic response to PLM is transient in nature lasting $<45 \mathrm{~s}$ in the SCI population. Thus, the aim of this investigation was to determine whether this transient hyperemic response is repeatable when bouts of PLM are interspersed with 1 min recovery periods. Not only are we the first to report that the response is repeatable, but also the hyperemic response appears to be more constant in this population when individuals are in a more aggressive supine position. This 
further supports the use of PLM to improve vascular health and tissue perfusion. The bouts of PLM also generated robust increases in SBF and reduced blood pooling, which could aid in lessening the impact of pressure ulcers and DVTs. Additional studies should be performed to determine the optimum PLM duration and rest period and measure markers of vascular health following chronic PLM.

Acknowledgements This work was supported by SPIRE award 1121 RX001732-01A1 from the United States Department of Veterans Affairs Rehabilitation Research and Development Service. The contents do not represent the views of the U.S. Department of Veterans Affairs or the United States Government

\section{Compliance with ethical standards}

Conflict of interest The authors declare that they have no conflict of interest.

\section{References}

1. Bogie KM, Wang X, Triolo RJ. Long-term prevention of pressure ulcers in high-risk patients: a single case study of the use of gluteal neuromuscular electric stimulation. Arch Phys Med Rehabil. 2006;87:585-91.

2. Phillips WT, Kiratli BJ, Sarkarati M, Weraarchakul G, Myers J, Franklin B, et al. Effect of spinal cord injury on the heart and cardiovascular fitness. Curr Probl Cardiol. 1998;23:641-716.

3. de Groot PC, Hjeltnes N, Heijboer AC, Stal W, Birkeland K. Effect of training intensity on physical capacity, lipid profile and insulin sensitivity in early rehabilitation of spinal cord-injured individuals. Spinal Cord. 2003;41:673-9.

4. Hopman MT, WNJC VanAsten, Oeseburg B. Changes in blood flow in the common femoral artery related to inactivity and muscle atrophy in individuals with long-standing paraplegia. Oxyg Transp Tissue. 1996;388:379-83.

5. Martin TP, Stein RB, Hoeppner PH, Reid DC. Influence of electrical stimulation on the morphological and metabolic properties of paralyzed muscle. J Appl Physiol. 1992;72:1401-6.

6. McDaniel J, Fjeldstad AS, Ives SJ, Hayman MA, Kithas P, Richardson RS. Central and peripheral contributors to skeletal muscle hyperemia: response to passive limb movement. J Appl Physiol. 2010;108:76-84.

7. Herr MD, Imadojemu V, Kunselman AR, Sinoway LI. Characteristics of the muscle mechanoreflex during quadriceps contractions in humans. J Appl Physiol. 1999;86:767-72.

8. Laughlin MH. Skeletal muscle blood flow capacity: role of muscle pump in exercise hyperemia. Am J Physiol. 1987;253: H993-1004.

9. Groot HJ, Trinity JD, Layec G, Rossman MJ, Ives SJ, Morgan $\mathrm{DE}$, et al. The role of nitric oxide in passive leg movementinduced vasodilatation with age: insight from alterations in femoral perfusion pressure. J Physiol. 2015;593:3917-28.

10. Clifford PS, Kluess HA, Hamann JJ, Buckwalter JB, Jasperse JL. Mechanical compression elicits vasodilatation in rat skeletal muscle feed arteries. J Physiol. 2006;572:561-7.

11. Ballaz L, Fusco N, Crétual A, Langella B, Brissot R. Acute peripheral blood flow response induced by passive leg cycle exercise in people with spinal cord injury. Arch Phys Med Rehabil. 2007;88:471-6.
12. Venturelli M, Amann M, Layec G, McDaniel J, Trinity JD, Fjeldstad AS, et al. Passive leg movement-induced hyperaemia with a spinal cord lesion: evidence of preserved vascular function. Acta Physiol. 2014;210:429-39.

13. Venturelli M, Amann M, McDaniel J, Trinity JD, Fjeldstad AS, Richardson RS. Central and peripheral hemodynamic responses to passive limb movement: the role of arousal. Am J Physiol Heart Circ Physiol. 2012;302:H333-9.

14. Ter Woerds W, De Groot PC, van Kuppevelt DH, Hopman MT. Passive leg movements and passive cycling do not alter arterial leg blood flow in subjects with spinal cord injury. Phys Ther. 2006;86:636-45.

15. Svensson M, Siösteen A, Wetterqvist H, Sullivan L. Influence of physiotherapy on leg blood flow in patients with complete spinal cord injury lesions. Physiother Theory Pract. 1995;11:97-107.

16. Hellsten Y, Rufener N, Nielsen JJ, Høier B, Krustrup P, Bangsbo J. Passive leg movement enhances interstitial VEGF protein, endothelial cell proliferation, and eNOS mRNA content in human skeletal muscle. Am J Physiol Regul Integr Comp Physiol. 2008;294:R975-R982.

17. Ferrari M, Mottola L, Quaresima V. Principles, techniques, and limitations of near infrared spectroscopy. Can J Appl Physiol. 2004;29:463-87.

18. Bonner RF, Nossal R. Principles of laser-Doppler flowmetry. In: Shepherd AP, Öberg PA, editors. Laser-Doppler blood flowmetry. US: Springer; 1990, pp. 17-45.

19. Bentzer P, Nielsen N, Arner M, Danielsen N, Ekblad E, Lundborg $\mathrm{G}$, et al. Supersensitivity in rat micro-arteries after short-term denervation. Acta Physiol Scand. 1997;161:125-33.

20. McDaniel J, Hayman MA, Ives SJ, Fjeldstad AS, Trinity JD, Wray DW, et al. Attenuated exercise induced hyperaemia with age: mechanistic insight from passive limb movement. J Physiol. 2010;588:4507-17.

21. Trinity JD, McDaniel J, Venturelli M, Fjeldstad AS, Ives SJ, Witman MA, et al. Impact of body position on central and peripheral hemodynamic contributions to movement-induced hyperemia: implications for rehabilitative medicine. Am J Physiol Heart Circ Physiol. 2011;300:H1885-91.

22. Trinity JD, Groot JH, Layec G, Rossman MJ, Ives SJ, Morgan DE, et al. Passive leg movement and nitric oxide-mediated vascular function: The impact of age. Am J Physiol Heart Circ Physiol. 2015;308:H672-9.

23. Trinity JD, Groot HJ, Layec G, Rossman MJ, Ives SJ, Runnels S, et al. Nitric oxide and passive limb movement: a new approach to assess vascular function. J Physiol. 2012;590:1413-25.

24. Muraki S, Ehara Y, Yamasaki M. Cardiovascular responses at the onset of passive leg cycle exercise in paraplegics with spinal cord injury. Eur J Appl Physiol. 2000;81:271-4.

25. Trinity JD, Amann M, McDaniel J, Fjeldstad AS, Barrett-O'Keefe $\mathrm{Z}$, Runnels S, et al. Limb movement-induced hyperemia has a central hemodynamic component: evidence from a neural blockade study. Am J Physiol Heart Circ Physiol. 2010;299: H1693-700.

26. Carmeliet P. Mechanisms of angiogenesis and arteriogenesis. Nat Med. 2000;6:389-96.

27. Liu Y, Figley S, Spratt SK, Lee G, Ando D, Surosky R, et al. An engineered transcription factor which activates VEGF-A enhances recovery after spinal cord injury. Neurobiol Dis. 2010;37:384-93.

28. Claydon VE, Steeves JD, Krassioukov A. Orthostatic hypotension following spinal cord injury: understanding clinical pathophysiology. Spinal Cord. 2005;44:341-51.

29. Kinzer SM, Convertino VA. Role of leg vasculature in the cardiovascular response to arm work in wheelchair-dependent populations. Clin Physiol. 1989;9:525-33.

30. Miranda AR, Hassouna HI. Mechanisms of thrombosis in spinal cord injury. Hematol Oncol Clin North Am. 2000;14:401-16. 
31. Lensing AWA, Prandoni P, Prins M, Büller HR. Deep-vein thrombosis. Lancet. 1999;353:479-85.

32. Sonenblum SE, Vonk TE, Janssen TW, Sprigle SH. Effects of wheelchair cushions and pressure relief maneuvers on ischial interface pressure and blood flow in people with spinal cord injury. Arch Phys Med Rehabil. 2014;95:1350-7.

33. Coggrave MJ, Rose LS. A specialist seating assessment clinic: changing pressure relief practice. Spinal Cord. 2003;41:692-5.
34. Garshick E, Kelley A, Cohen SA, Garrison A, Tun CG, Gagnon $\mathrm{D}$, et al. A prospective assessment of mortality in chronic spinal cord injury. Spinal Cord. 2005;43:408-16.

35. DeVivo MJ, Black KJ, Stover SL. Causes of death during the first 12 years after spinal cord injury. Arch Phys Med Rehabil. 1993;74:248-54.

36. Burkett LN, Chisum J, Pierce J, Pomeroy K, Fisher J, Martin M. Blood flow and lactic acid levels in exercising paralyzed wheelchair bound individuals. Adapt Phys Act Q. 1988;5:60-73. 